\title{
Weekly injection of IL-2 using an injectable hydrogel reduces autoimmune diabetes incidence in NOD mice
}

\author{
Nadine Nagy ${ }^{1}$ (D) $\cdot$ Gernot Kaber $^{1}$ (D) - Michael J. Kratochvil ${ }^{1,2}$ (D) $\cdot$ Hedwich F. Kuipers ${ }^{1}$ (D) Shannon M. Ruppert $^{1} \cdot$ \\ Koshika Yadava ${ }^{1}$ (D) - Jason Yang ${ }^{1}$ (D) - Sarah C. Heilshorn ${ }^{2}$ (D) - S. Alice Long ${ }^{3}$ (D) - Alberto Pugliese ${ }^{4}$ (D) \\ Paul L. Bollyky ${ }^{1}$ (it)
}

Received: 15 May 2020 / Accepted: 25 August 2020 / Published online: 30 October 2020

(C) Springer-Verlag GmbH Germany, part of Springer Nature 2020

\begin{abstract}
Aims/hypothesis IL-2 injections are a promising therapy for autoimmune type 1 diabetes but the short half-life of this cytokine in vivo limits effective tissue exposure and necessitates frequent injections. Here we have investigated whether an injectable hydrogel could be used to promote prolonged IL-2 release in vivo.

Methods Capitalising on the IL-2-binding capabilities of heparin, an injectable hydrogel incorporating clinical-grade heparin, collagen and hyaluronan polymers was used to deliver IL-2. The IL-2-release kinetics and in vivo stability of this material were examined. The ability of soluble IL-2 vs hydrogel-mediated IL-2 injections to prevent autoimmune diabetes in the NOD mouse model of type 1 diabetes were compared.

Results We observed in vitro that the hydrogel released IL-2 over a 12-day time frame and that injected hydrogel likewise persisted 12 days in vivo. Notably, heparin binding potentiates the activity of IL-2 and enhances IL-2- and TGF $\beta$-mediated expansion of forkhead box P3-positive regulatory T cells (FOXP3 ${ }^{+}$Tregs). Finally, weekly administration of IL-2-containing hydrogel partially prevented autoimmune diabetes while injections of soluble IL-2 did not.

Conclusions/interpretation Hydrogel delivery may reduce the number of injections required in IL-2 treatment protocols for autoimmune diabetes.
\end{abstract}

Keywords Autoimmune $\cdot$ Controlled release $\cdot$ Diabetes $\cdot$ Heparin $\cdot$ Hyaluronan $\cdot$ Hydrogels $\cdot$ IL-2 $\cdot$ Treg

\begin{tabular}{|c|c|}
\hline \multicolumn{2}{|c|}{ Abbreviations } \\
\hline FOXP3 & Forkhead box P3 \\
\hline HA & Hyaluronan \\
\hline HI & Heparin \\
\hline LN & Lymph node \\
\hline Tconv & Conventional $\mathrm{T}$ cell \\
\hline Treg & $\mathrm{FOXP}^{+}{ }^{+}$regulatory $\mathrm{T}$ cell \\
\hline
\end{tabular}

Nadine Nagy

nnagy@stanford.edu

1 Division of Infectious Diseases and Geographic Medicine, Department of Medicine, Stanford University School of Medicine, Stanford, CA, USA

2 Department of Materials Science and Engineering, Stanford University, Stanford, CA, USA

3 Benaroya Research Institute, Seattle, WA, USA

4 Diabetes Research Institute, Leonard M. Miller School of Medicine, University of Miami, Miami, FL, USA

\section{Introduction}

Type 1 diabetes is characterised by the progressive immune cell-mediated destruction of pancreatic beta cells and the failure of regulatory mechanisms that normally prevent this, including regulatory $\mathrm{T}$ cells (Tregs) [1].

One critical factor that governs Treg function is the cytokine IL-2 [2]. The potential role of low-dose IL-2 in type 1 diabetes is a subject of active exploration and a frontier in the treatment and prevention of autoimmunity [3]. Low-dose IL-2 affects two critical cell populations of the immune system: $\mathrm{T}$ cells and natural killer (NK) cells. However, IL-2 has a short half-life, depending on the route of administration, from $7 \mathrm{~min}$ for i.v. to several hours for i.p. and s.c. administration $[4,5]$. In addition, the dosing protocols vary depending on the disease. Cancer treatment usually requires daily dosing [6], whereas autoimmune disease protocols are variable, with some calling for administration of doses spaced at 


\section{Research in context}

\section{What is already known about this subject?}

- $\quad \mathrm{IL}-2$ is a promising therapy for type 1 diabetes and other autoimmune diseases

- The use of IL-2 alone is unlikely to prevent autoimmune diabetes

- Despite the therapeutic potential of IL-2 injections, the short half-life of IL-2 in vivo limits effective tissue exposure to IL-2. This necessitates frequent injections and may impact efficacy

\section{What is the key question?}

- Is it possible to increase tissue exposure to IL-2 and reduce the risk of autoimmune type 1 diabetes progression using a hydrogel?

\section{What are the new findings?}

- We have developed an injectable hydrogel that incorporates the polymer heparin to enable the sustained release of IL-2

- The hydrogel releases IL-2 over a 12-day period and upon injection is stable for this long. The hydrogel promotes an increase in regulatory $T$ cells (Tregs) in vivo, which play a crucial role in autoimmune type 1 diabetes

- In the NOD mouse model of type 1 diabetes, once-weekly administration of a hydrogel containing IL-2 and hyaluronan/heparin/collagen partially prevented diabetes while weekly injections of soluble IL-2 did not

How might this impact on clinical practice in the foreseeable future?

- Hydrogels may allow less frequent IL-2 injections and facilitate protocols designed to prevent type 1 diabetes

as much as 5 day intervals [6, 7]. It would be desirable to extend the effective half-life of IL-2 to administer as few injections as possible.

One matrix component that has been used to bind and deliver cytokines in a sustained manner is heparin [8]. Heparin binding also influences the bioactivity of many cytokines, including IL-2 [9-12], although effects on Tregs have not been previously examined.

We hypothesised that it would be possible to adapt a heparin-based hydrogel for the sustained and localised release of IL-2 to reduce autoimmune diabetes in the NOD mouse model of type 1 diabetes.

\section{Methods}

Mice All animals were bred and maintained under specific pathogen-free conditions with free access to food and water in the vivarium at Stanford University (Stanford, CA). Female NOD and C57BL/6 mice were purchased from the Jackson Laboratory (Bar Harbor, ME, USA). All experiments and animal use procedures were approved by the Animal Care \& Use Committee of Stanford University. The animal assignment to experimental groups was performed randomly. Due to multiple different treatments during the course of the experiments, the experimenters were not blinded to group assignments, but experimenters were blinded for analysis.
Weight and diabetes monitoring Beginning at 4 weeks of age, mice were weighed and bled weekly to measure their blood glucose levels. When two consecutive blood glucose readings of $13.87 \mathrm{mmol} / 1$ or greater were recorded, mice were considered diabetic.

Hydrogels The hydrogel system is made up of thiolated hyaluronan/heparin/collagen components crosslinked with polyethylene glycol diacrylate. The heparin is a low-molecular-weight, clinical-grade product. The hyaluronan is a standardised, clinical-grade $500 \mathrm{kDa}$ product. The collagen is type 1 collagen and is also clinical grade. These gel components together make up $1 \%$ weight/volume of the hydrogel. The Extracel and Extracel-HP are commercially available preparations that have been widely reported in the literature [13-15]. Extracel (hyaluronan/collagen) and Extracel-HP (hyaluronan/heparin/collagen) hydrogels (BioTime, Alameda, CA, USA) were generated as per the manufacturer's instructions. To assess the stability of these in vivo, $200 \mu \mathrm{l}$ hydrogel incorporating an Alexa Fluor 790 fluorescent tag (Thermo Fisher, Waltham, MA, USA) were injected s.c. or i.p. into mice and allowed to polymerise in situ. Residual hydrogel mass was assessed at multiple days post injection using an IVIS 100 in vivo imaging system (Perkin Elmer, Waltham, MA, USA). A fibrin hydrogel was formulated by cleaving $5 \mathrm{mg} / \mathrm{ml}$ salmon fibrinogen (SeaRun Holdings, Freeport, ME, USA) with $2 \mathrm{U} / \mathrm{ml}$ salmon thrombin (SeaRun Holdings) in Extracel. A matrigel hydrogel 
(Corning, Corning, NY, USA) was prepared according to manufacturer's instruction.

IL-2 The IL-2 used in this study was Proleukin (aldesleukin; Clinigen, PA, USA). It is a human recombinant IL-2 product, a highly purified protein with a molecular weight of approximately $15,300 \mathrm{Da}$. This is a clinical-grade product that has been used widely for over 20 years in numerous animal models and human clinical trials $[6,16]$.

IL-2 in vivo studies For one set of experiments, 6-week-old NOD mice were treated with either 25,000 IU IL-2 once weekly or the same amount of IL-2 delivered in the context of a single hydrogel injection, or PBS injections as a negative control. After 1 month, mice were killed, mesenteric lymph nodes (LNs) were collected, and populations of lymphocytes were assessed by flow cytometry. For another set of experiments the above-mentioned treatment was administered to mice from 6 to 21 weeks of age. The mice were subsequently monitored for diabetes onset until 33 weeks of age.

Measurement of IL-2 release from hydrogels Hydrogels were cast into a 96 well plate, $100 \mu \mathrm{l}$ per well. After the hydrogels were formed, $200 \mu \mathrm{l}$ of $1600 \mathrm{IU}$ IL-2/ml PBS was added per well and incubated overnight. The loading solution was collected for later analysis. Release was conducted by incubating the samples in $200 \mu \mathrm{l}$ of PBS, which was collected and replaced at the specified time points. IL-2 concentration was measured using an IL-2 ELISA (BioLegend, San Diego, CA, USA).

IL-2 proliferation assay CTLL2 cells (ATCC, Manassas, VA, USA), were cultured for $48 \mathrm{~h}$ in the context of increasing concentrations of IL-2 with or without heparin. Proliferation was measured using a resazurin-based Reliablue Cell Viability Reagent (ATCC, Manassas, VA, USA).

Isolation and analysis of leucocyte populations Total leukocytes were isolated from lymph nodes from 10-week-old NOD mice, as previously described [17]. $\mathrm{CD}^{+} \mathrm{T}$ cells were isolated from the pooled cell suspensions using an EasySep Mouse $\mathrm{CD}^{+}{ }^{+} \mathrm{T}$ Cell Isolation Kit (Stemcell, Vancouver, BC, Canada), following the manufacturer's instructions. For T cell activation and forkhead box P3-positive (FOXP3 ${ }^{+}$) induction studies, cell culture plates (96-well) were coated overnight with $5 \mu \mathrm{g} / \mathrm{ml}$ antiCD3 antibody (catalogue no. 145-2C11; BD Biosciences, San Jose, CA, USA) and $2.5 \mu \mathrm{g} / \mathrm{ml}$ anti-CD28 antibody (catalogue no. 37.51; BD Biosciences). Subsequently, $1 \times 10^{5}$ cells were cultured with or without soluble $100 \mathrm{IU} / \mathrm{ml} \mathrm{IL-2} \mathrm{(Proleukin}$ [aldesleukin]; Clinigen) and $50 \mathrm{ng} / \mathrm{ml} \mathrm{TGF} \beta$ (catalogue no. 21C11; BioLegend), as indicated using previously published protocols [18]. Cells were stained and flow cytometry was performed as previously described [17]. Analysis was done on an LSR II instrument (BD Biosciences) in the Stanford Shared
FACS Facility (Stanford, CA, USA). Analysis was carried out on Flowjo v10 (Ashland, OR, USA).

Statistical analysis Data are expressed as means \pm SEM of $n$ independent measurements. A $p$ value of $<0.05$ was considered significant. Significance of the difference between the means of two or three groups of data was evaluated using a two-tailed $t$ test or one-way ANOVA with Šidák's multiple comparisons post-test.

\section{Results}

Heparin potentiates IL-2 Treg induction While heparin is reported to enhance the activity of IL-2, we investigated whether heparin-bound IL-2 likewise potentiates the effects of the cytokine on Tregs. In vitro we observed that proliferation with heparin complexed with IL-2 was more than twofold that with IL-2 alone (Fig. 1a). We then sought to determine whether heparin complexed with IL-2 likewise enhances the impact of IL-2 on Treg induction from $\mathrm{CD}^{+} \mathrm{GFP} / \mathrm{FOXP} 3^{-}$ conventional $\mathrm{T}$ cells (Tconv) precursors activated with antiCD3 and anti-CD28 in the presence of TGF $\beta$ and IL-2 (Fig. 1b) [19]. The presence of heparin and IL-2 greatly increased Treg induction in this assay (Fig. 1c).

Heparin- and IL-2 containing hydrogels potentiates Treg induction We next studied whether heparin in the context of a hydrogel also potentiates the effects of IL-2 on Treg induction. To this end, we coated tissue culture plates with a commercially available hyaluronan/heparin/collagen hydrogel preparation (Fig. 1d) and used the same induction protocol (Fig. 1e). In the presence of IL-2, the hyaluronan/heparin/ collagen hydrogel significantly increased Treg induction vs the non-hydrogel-containing condition (Fig. 1f). Notably, repeating the experiment with a hyaluronan/collagen hydrogel lacking the heparin component, a fibrin hydrogel or a matrigel hydrogel did not potentiate Treg induction (data not shown).

Together, these data indicate that heparin potentiates the effects of IL-2 on Treg expansion, and that this stimulus can be delivered as a hydrogel.

Hydrogels release IL-2 in a sustained manner We next sought to quantify the capacity of hydrogels to bind and slowly release IL-2 over time. The hyaluronan/heparin/collagen hydrogel eluted IL-2 through day 12, while the hyaluronan/ collagen hydrogel eluted IL-2 through day 7 (Fig. 1g,h). These data indicate that hydrogels retain IL-2, releasing it over time, and that heparin enhances this.

Hydrogel persists in vivo To determine whether the hyaluronan/heparin/collagen hydrogel is stable when injected in vivo, the hydrogel was delivered via s.c. and i.p. injection 
a

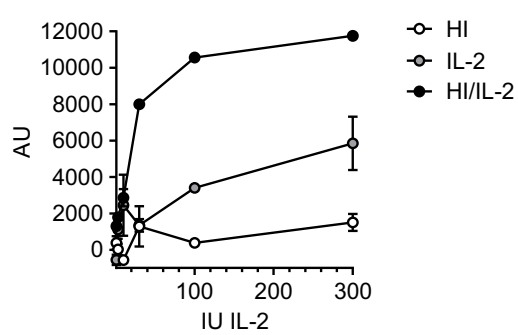

e

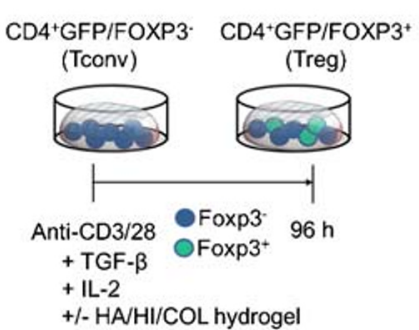

i

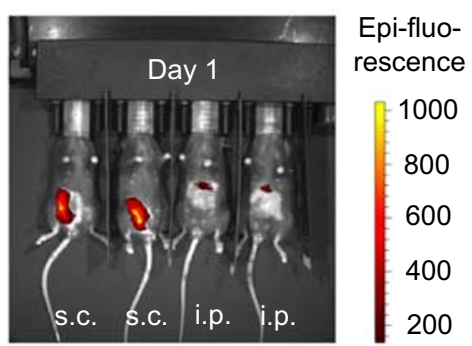

b

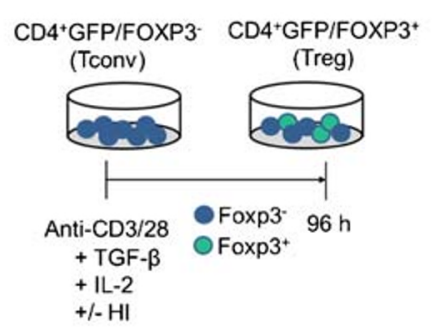

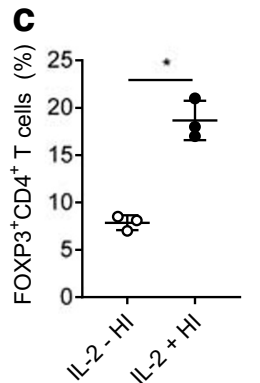

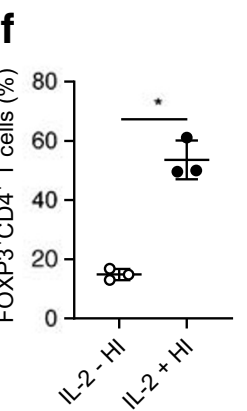

d

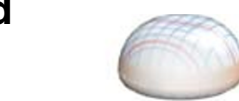

- Thiol-modified denatured collagen

- Thiol-modified hyaluronan

- Thiol-modified heparin

- Polyethylene glycol diacrylate + IL-2
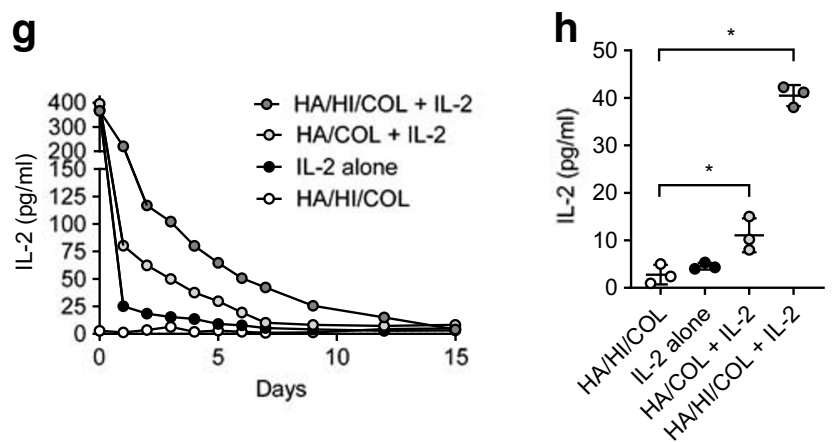

k

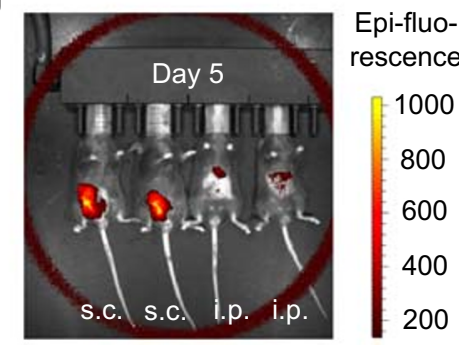

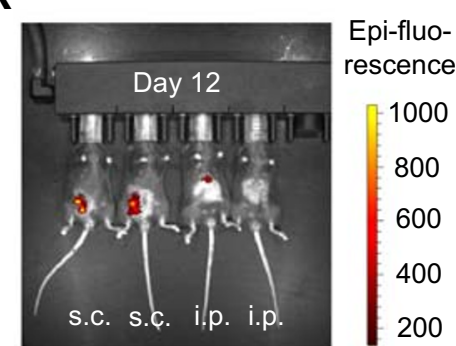

Fig. 1 Hydrogels release IL-2 in a sustained manner and potentiate Treg induction in the presence of heparin (HI). (a) CTLL2 cells were cultured in the presence of increasing concentrations of IL-2 with or without HI. Proliferation was measured by resazurin incorporation and expressed as arbitrary units (AU). Data are shown for triplicate wells per condition. (b) Schematic of $\mathrm{CD}^{+} \mathrm{GFP} / \mathrm{FOXP}^{-} \mathrm{T}$ cells isolated from healthy (nondiabetic) mice, cultured in the setting of anti-CD3 and anti-CD28, TGF $\beta$ and IL- 2 in the absence or presence of HI. (c) Percentage of $\mathrm{CD}^{+}{ }^{+} \mathrm{T}$ cells that were $\mathrm{FOXP}^{+}$from the experiment described in (b). Quantification of $n=3$ identical experiments. Data represent mean \pm SEM; $* p<0.05$ as determined by a two-sided $t$ test. (d) Schematic of hydrogel composition. (e) Schematic of $\mathrm{CD}^{+}{ }^{+} \mathrm{GFP} / \mathrm{FOXP}^{-} \mathrm{T}$ cells, isolated from healthy (non-diabetic) mice cultured in the setting of anti$\mathrm{CD} 3 /-\mathrm{CD} 28, \mathrm{TGF} \beta$ and IL-2 in the absence or presence of $\mathrm{HI}$ and

and allowed to polymerise in situ. Residual hydrogel mass in the mice was then assessed using the IVIS in vivo imaging system at 1, 5 and 12 days post hydrogel injection (Fig. 1i-k). Injection of the hydrogel s.c. resulted in longer persistence, which was still clearly seen at day 12 post injection (Fig. 1k). In general, hydrogel introduced via i.p. injection did not show a strong signal using the IVIS in vivo imaging (Fig. 1i-k). These data demonstrate that a hyaluronan/heparin/collagen hydrogel polymerises in vivo and is stable for up to 12 days. hydrogel. COL, collagen; HA, hyaluronan. (f) Percentage of CD4 ${ }^{+} \mathrm{T}$ cells that were $\mathrm{FOXP}^{+}$from experiment described in (e). Quantification of $n=3$ identical experiments. Data represent mean $\pm \mathrm{SEM}$; $* p<0.05$ as determined by a two-sided $t$ test. (g) Hydrogel IL-2 release curve for different hydrogel compositions (HA, HI, COL and/or IL-2). (h) IL-2 release from hydrogels measured by IL-2 ELISA at day 7. Data represent mean $\pm \mathrm{SEM} ; * p<0.05$ as determined by ANOVA followed by Šidák's multiple comparisons test. Data in $(\mathbf{g})$ and (h) are shown for the mean of triplicate wells per condition. (i-k) Hydrogels of $50 \mu \mathrm{l}$ volume incorporating an Alexa Fluor 790 fluorescent tag were injected into mice s.c. and i.p. and allowed to polymerise in situ. Residual hydrogel mass was then assessed at 1 (i), 5 (j) and 12 days (k) post injection using an IVIS in vivo imaging system. Data are representative of three independent experiments

Hydrogel-mediated IL-2 delivery is associated with an in vivo Treg increase To investigate whether hydrogel-mediated IL-2 delivery could be used to promote Treg expansion in vivo, NOD mice were treated by once weekly i.p. injection of 25,000 IU IL-2 either in its soluble form or in a hyaluronan/ heparin/collagen hydrogel. After 1 month of treatment, the mice were killed and $\mathrm{T}$ cell populations in the mesenteric LNs were then assessed via flow cytometry.

We observed that the percentage of $\mathrm{CD} 3^{+} \mathrm{CD} 4^{+} \mathrm{T}$ cells and $\mathrm{CD} 3^{+} \mathrm{CD} 8^{+} \mathrm{T}$ cells in the LNs was significantly decreased in 

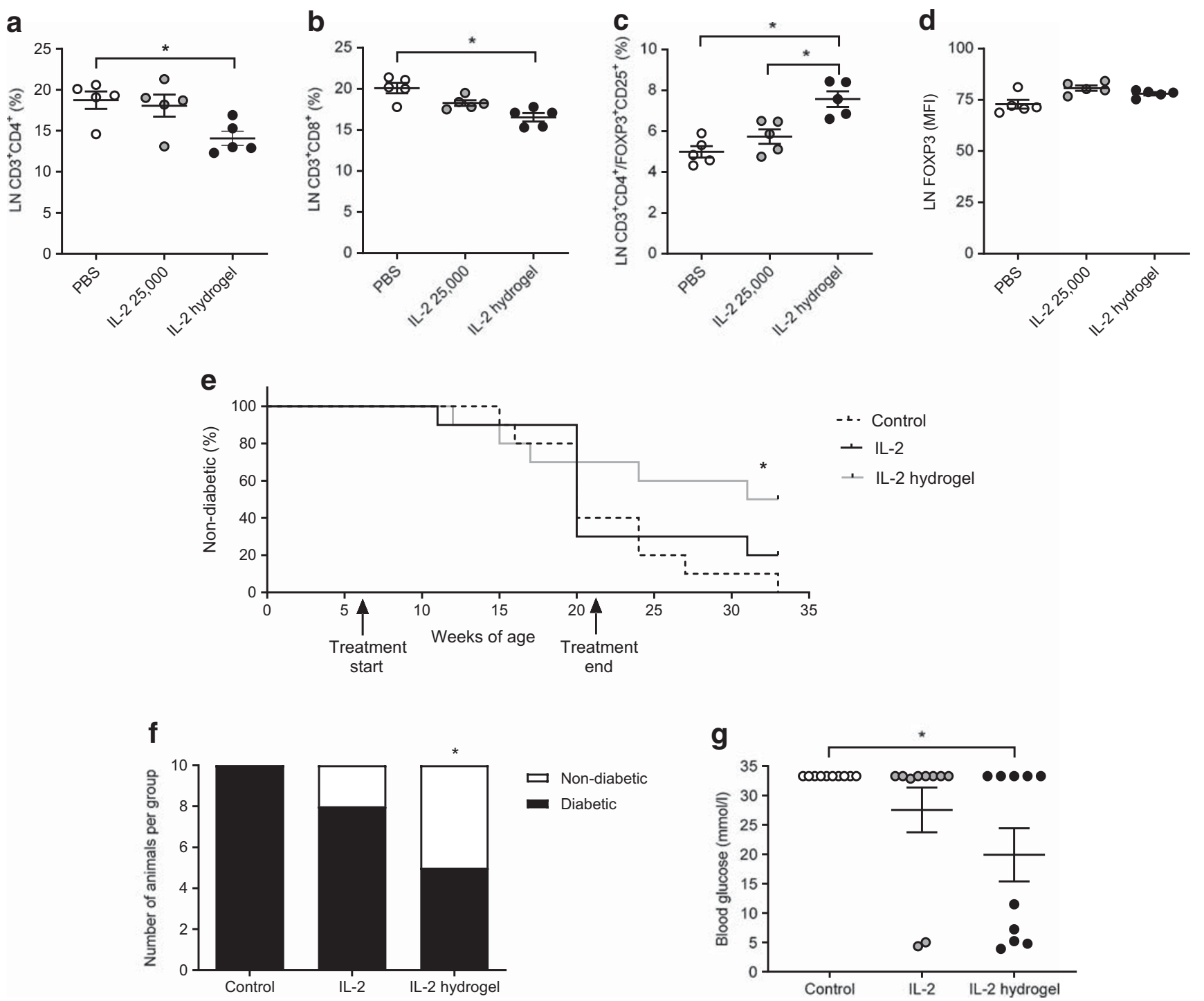

Fig. 2 In vivo heparin (HI) hydrogels are associated with an increase in $\mathrm{FoxP}^{+}$Tregs and reduce diabetes onset in NOD mice. (a-d) Six-weekold NOD mice were treated with either 25,000 IU IL-2 once weekly or the same amount of IL-2 delivered in the context of a single hydrogel injection, or PBS injections as a negative control. After 1 month, mice were killed, mesenteric LNs were collected, and populations of lymphocytes were assessed by flow cytometry. In particular, the percentage of $\mathrm{CD}^{+} \mathrm{CD}^{+} \mathrm{T}$ cells $(\mathbf{a})$, the percentage of $\mathrm{CD}^{+} \mathrm{CD}^{+} \mathrm{T}$ cells $(\mathbf{b})$, $\mathrm{CD}^{+} \mathrm{CD}^{+} / \mathrm{FOXP} 3{ }^{+} \mathrm{CD} 25^{+}$Tregs (c) and FOXP 3 mean fluorescence intensity (MFI) (d) were assessed. Data are representative of two independent experiments; $n=5$ mice per group. Data represent mean $\pm \mathrm{SEM}$;

the IL-2 hydrogel treatment group (Fig. 2a,b). The percentage of $\mathrm{CD}^{+}{ }^{+} \mathrm{CD} 4^{+} \mathrm{FOXP}^{+}$Tregs was significantly increased in the setting of the IL-2 hydrogel group (Fig. 2c), while the FOXP3 mean fluorescence intensity (MFI) was unchanged by either treatment (Fig. 2d). Together, these data indicate that hydrogel-mediated IL-2 delivery increases Tregs in vivo.

\section{IL-2 delivery via hydrogel reduces the incidence of diabetes onset in NOD mice In parallel to studying the effects of the IL-}

$* p<0.05$ as determined by ANOVA followed by Šidák's multiple comparisons test. (e) Starting at 6 weeks of age, NOD mice received either 25,000 IU IL-2 once weekly, or the same amount of IL-2 delivered in the context of a once a week hydrogel injection. PBS injections served as negative controls. Mice were then monitored weekly for diabetes onset. This treatment was administered to mice from 6 to 21 weeks of age and mice were subsequently monitored until 33 weeks of age. (f, g) Number of diabetic mice per group (f) and blood glucose (g) at 33 weeks of age, 3 months after treatment ended. Data represent mean $\pm \mathrm{SEM}, n=10$ mice per group. ${ }^{*} p<0.05$ as determined by ANOVA followed by Šidák's multiple comparisons test

2 hydrogel on Treg expansion, we also investigated whether this treatment regimen could prevent autoimmune diabetes in NOD mice.

NOD mice were i.p. injected once weekly with $25,000 \mathrm{IU}$ IL-2 either in its soluble form or in a hyaluronan/heparin/ collagen hydrogel. This treatment was administered to 6week-old mice for 15 weeks, a Kaplan-Meier curve shows the percentage of non-diabetic mice in the different treatment groups over time (Fig. 2e). Three months later, at 33 weeks of 
age, all mice in the control group, eight out of ten in the IL-2 group, and five out of ten in the IL-2 hydrogel group were diabetic (Fig. 2f). At 33 weeks of age, the blood glucose values of the control mice were $>33.33 \mathrm{mmol} / 1$, $\sim 27.75 \mathrm{mmol} / 1$ for the IL-2 treatment group and $\sim 20.53 \mathrm{mmol} / 1$ for the IL-2 hydrogel group (Fig. $2 \mathrm{~g}$ ). These data suggest that once weekly hydrogel delivery of IL-2 could partially prevent autoimmunity in this animal model.

\section{Discussion}

We report that once weekly injections of a hydrogel containing heparin and IL-2 promote Treg expansion and partially prevent autoimmunity in the NOD mouse model of type 1 diabetes. In contrast, the same IL-2 regimen injected without hydrogel did not. Other groups have used protocols in which IL-2 was administered daily or several times a week [20-23]; these different treatment regimens make it difficult to compare data between studies. Our data suggest that it may be possible to reduce the likelihood of onset of autoimmune diabetes with less frequent injections using IL-2-releasing hydrogel preparations. Currently, IL2 use alone cannot be used in humans to prevent autoimmune diabetes; we used an exploratory approach in our heparin- and IL-2-containing hydrogel study.

We have demonstrated that heparin delivered in diverse ways, alone or in the form of a hydrogel, amplifies the impact of IL-2 on multiple cell types. We report that a hydrogel containing heparin can release IL-2 over time using an assay performed in the absence of cells that does not therefore involve cellular responses to IL-2. Thus, we conclude that heparin has effects both on the potency of IL-2 as well as its sustained release from a hydrogel over time. We believe that enhanced IL-2 activity, as well as the extended release of IL-2 through very slow diffusion and degradation of the hydrogel itself, contribute to the effects reported here.

This hydrogel may have potential in treating other autoimmune disorders that are responsive to IL-2 [24], perhaps particularly autoimmune diseases of the skin. However, the safety and tolerability of hydrogel materials in the context of IL-2 will need to be examined [25, 26]. Moreover, it would be important to evaluate the performance of these hydrogel materials over a range of IL-2 concentrations and formulations. Based on our findings, we conclude that hydrogel-mediated IL-2 delivery may be a useful approach for delivering IL-2 therapeutically.

Acknowledgements We thank N. L. Haddock (Department of Medicine, Stanford University, Stanford, CA, USA) for her assistance with drafting the revised manuscript and for generating the graphical abstract.

Data availability The data are available from the corresponding author.
Funding This work was supported in part by the National Institutes of Health (NIH) grants R01 DK096087-01, R01 HL113294-01A1, R01 DK116782-01A1 and U01 AI101984 to PLB. KY was supported by the Swiss National Science Foundation early postdoctoral mobility grant and the Stanford Child Health Research Institute and the Stanford NIHNCATS-CTSA (grant no. UL1 TR001085). This work was also supported by grants from the JDRF 3-PDF-2014-224-A-N to NN and 1-SRA2018-518-S-B Innovation Award to PLB and by grants from the Harrington Institute, Stanford SPARK, the Stanford Child Health Research Institute, all to PLB.

Authors' relationships and activities The authors declare that there are no relationships or activities that might bias, or be perceived to bias, their work.

Contribution statement NN, GK and PLB designed the study. NN, GK, MJK, HFK, SMR, KY and JY analysed the data. All authors interpreted the results. NN and PLB drafted the first version of the manuscript. All authors critically revised earlier versions of the manuscript. All authors read and approved the final version of the manuscript to be published. PLB is the guarantor of this work.

\section{References}

1. Bollyky PL, Falk BA, Long SA et al (2009) CD44 costimulation promotes $\mathrm{FoxP}^{+}$regulatory $\mathrm{T}$ cell persistence and function via production of IL-2, IL-10, and TGF-beta. J Immunol 183(4): 2232-2241. https://doi.org/10.4049/jimmunol.0900191

2. Bayer AL, Yu A, Malek TR (2007) Function of the IL-2R for thymic and peripheral $\mathrm{CD} 4{ }^{+} \mathrm{CD} 25+$ Foxp $3+\mathrm{T}$ regulatory cells. J Immunol 178(7):4062-4071. https://doi.org/10.4049/jimmunol. 178.7.4062

3. Rosenzwajg M, Churlaud G, Mallone R et al (2015) Low-dose interleukin-2 fosters a dose-dependent regulatory $\mathrm{T}$ cell tuned milieu in T1D patients. J Autoimmun 58:48-58. https://doi.org/ 10.1016/j.jaut.2015.01.001

4. Lotze MT, Frana LW, Sharrow SO, Robb RJ, Rosenberg SA (1985) In vivo administration of purified human interleukin 2. I. Half-life and immunologic effects of the Jurkat cell line-derived interleukin 2. J Immunol 134(1):157-166

5. Cheever MA, Thompson JA, Kern DE, Greenberg PD (1985) Interleukin 2 (IL 2) administered in vivo: Influence of IL 2 route and timing on T cell growth. J Immunol 134:3895-3900 https:// www.jimmunol.org/content/134/6/3895

6. Jeal W, Goa KL (1997) Aldesleukin (recombinant interleukin-2): a review of its pharmacological properties, clinical efficacy and tolerability in patients with renal cell carcinoma. BioDrugs 7(4):285317. https://doi.org/10.2165/00063030-199707040-00005

7. Tahvildari M, Dana R (2019) Low-dose IL-2 therapy in transplantation, autoimmunity, and inflammatory diseases. J Immunol 203(11):2749-2755. https://doi.org/10.4049/jimmunol.1900733

8. Xu D, Esko JD (2014) Demystifying heparan sulfate-protein interactions. Annu Rev Biochem 83(1):129-157. https://doi.org/10. 1146/annurev-biochem-060713-035314

9. Yayon A, Klagsbrun M, Esko JD, Leder P, Ornitz DM (1991) Cell surface, heparin-like molecules are required for binding of basic fibroblast growth factor to its high affinity receptor. Cell 22 64(4): 841-848. https://doi.org/10.1016/0092-8674(91)90512-w

10. Wrenshall LE, Carlson A, Cerra FB, Platt JL (1994) Modulation of cytolytic T cell responses by heparan sulfate. Transplantation 57(7): 1087-1094. https://doi.org/10.1097/00007890-199404000-00018 
11. Najjam S, Mulloy B, Theze J, Gordon M, Gibbs R, Rider CC (1998) Further characterization of the binding of human recombinant interleukin 2 to heparin and identification of putative binding sites. Glycobiology 8(5):509-516. https://doi.org/10.1093/glycob/ 8.5.509

12. Ramsden L, Rider CC (1992) Selective and differential binding of interleukin (IL)-1a, IL-1b, IL-2 and IL-6 to glycosaminoglycans. Eur J Immunol 22:3027-3031. https://doi.org/10.1002/eji. 1830221139

13. Bollyky PL, Wu RP, Falk BA et al (2011) ECM components guide IL-10 producing regulatory T-cell (TR1) introduction from effector memory T-cell precursors. PNAS 108(19):7938-7943. https://doi. org/10.1073/pnas. 1017360108

14. Elia R, Fuegy PW, VanDelden A, Firpo MA, Prestwich GD, Peattie RA (2010) Stimulation of in vivo angiogenesis by in situ crosslinked, dual growth factor-loaded, glycosaminoglycan hydrogels. Biomaterials 31(17):4630-4638. https://doi.org/10. 1016/j.biomaterials.2010.02.043

15. Advanced BioMatrix (2020) HyStem: Thiol-Modified Hyaluronan Hydrogel Kit. Available from https://advancedbiomatrix.com/ hystem.html. Accessed 20 July 2020

16. Proleukin (2020) Proleukin (aldesleukin) recombinant IL-2. Available from: www.proleukin.com. Accessed 20 July 2020

17. Ruppert SM, Falk BA, Long SA, Bollyky PL (2015) Regulatory T cells resist cyclosporine-induced cell death via CD44-mediated signaling pathways. Int J Cell Biol 2015(4):614297-614210. https://oi.org/10.1155/2015/614297

18. Nagy N, Kaber G, Johnson PY et al (2015) Inhibition of hyaluronan synthesis restores immune tolerance during autoimmune insulitis. J Clin Invest 125(10):3928-3940. https://doi.org/10.1172/JCI79271

19. Walker MR, Kasprowicz DJ, Gersuk VH et al (2003) Induction of FoxP3 and acquisition of $\mathrm{T}$ regulatory activity by stimulated human
CD4 ${ }^{+}$CD25 ${ }^{-}$T cells. J Clin Invest 112(9):1437-1443. https://doi. org/10.1172/JCI19441

20. Serreze DV, Hamaguchi K, Leiter EH (1989) Immunostimulation circumvents diabetes in NODLt mice. J Autoimmun 2(6):759-776. https://doi.org/10.1016/0896-8411(89)90003-6

21. Rabinovitch A, Suarez-Pinzon WL, Shapiro AMJ, Rajotte RV, Power R (2002) Combination therapy with sirolimus and interleukin-2 prevents spontaneous and recurrent autoimmune diabetes in NOD mice. Diabetes 51(3):638-645. https://doi.org/ 10.2337/diabetes.51.3.638

22. Tang Q, Adams JY, Penaranda C et al (2008) Central role of defective interleukin-2 production in the triggering of islet autoimmune destruction. Immunity 28(5):687-697. https://doi.org/10.1016/j. immuni.2008.03.016

23. Grinberg-Bleyer Y, Baeyens A, You S et al (2010) IL-2 reverses established type 1 diabetes in NOD mice by a local effect on pancreatic regulatory T cells. J Exp Med 207(9):1871-1878. https://doi. org/10.1084/jem.20100209

24. Castela E, Le Duff F, Butori C et al (2014) Effects of low-dose recombinant interleukin 2 to promote T-regulatory cells in alopecia areata. JAMA Dermatol 150(7):748-751. https://doi.org/10.1001/ jamadermatol.2014.504

25. Long SA, Buckner JH, Greenbaum CJ (2013) IL-2 therapy in type 1 diabetes: "Trials" and tribulations. Clin Immunol 149(3):324-331. https://doi.org/10.1016/j.clim.2013.02.005

26. Long SA, Rieck M, Sanda S et al (2012) Rapamycin/IL-2 combination therapy in patients with type 1 diabetes augments Tregs yet transiently impairs $\beta$-cell function. Diabetes 61(9):2340-2348. https://doi.org/10.2337/db12-0049

Publisher's note Springer Nature remains neutral with regard to jurisdictional claims in published maps and institutional affiliations. 